\title{
THE
}

\section{Sociodemographic Disparities in Adult Child Informal Caregiving Intensity in the United States: Results from the New National Study of Caregiving}

Sarah K. Cook

Steven A. Cohen

University of Rhode Island, steven_cohen@uri.edu

Follow this and additional works at: https://digitalcommons.uri.edu/kinesiology_facpubs

The University of Rhode Island Faculty have made this article openly available.

Please let us know how Open Access to this research benefits you.

This is a pre-publication author manuscript of the final, published article.

Terms of Use

This article is made available under the terms and conditions applicable towards Open Access Policy Articles, as set forth in our Terms of Use.

\section{Citation/Publisher Attribution}

Cook S., Cohen S.(2018). Sociodemographic Disparities in Adult Child Informal Caregiving Intensity in the United States: Results from the New National Study of Caregiving. J Gerontol Nurs. 44(9) 15-20. doi: 10.3928/00989134-20180808-05

Available at: https://doi.org/10.3928/00989134-20180808-05 
Sociodemographic disparities in adult-child informal caregiving intensity in the US:

\section{2}

3

4 Sarah K. Cook ${ }^{1}$ and Steven A. Cohen ${ }^{2}$

5 1Vanderbilt Institute for Clinical and Translational Research, Nashville, Tennessee $7 \quad$ Rhode Island

\section{Results from the new National Study of Caregiving} ${ }^{2}$ Health Studies Program, Department of Kinesiology, University of Rhode Island, Kingston, Abstract: Nearly 60 million informal caregivers provide care to aging adults. Despite its many benefits, high intensity caregiving can impact caregiver health and quality-of-life. Therefore, the objective of our study was to assess socioeconomic and demographic disparities in caregiving intensity among informal caregivers. Using a randomized, nationally representative database of 1014 informal offspring caregivers from Medicare enrollment databases, the associations between informal caregiving intensity and age, race/ethnicity, and income were examined using binary and ordinal logistic regression. Caregiving intensity varied by demographics. High ADL caregiving was highest among Black, non-Hispanic caregivers. High IADL caregiving and high number of hours spent caregiving was highest in females, and non-White caregivers. Although the overall association between caregiving intensity and income was not significant, when stratified by race/ethnicity, this association was positive for White caregivers and negative for non-White caregivers. Health care providers frequently interact with informal caregivers and should be aware of trends in caregiving and the needs and supports available to ameliorate caregiver burden. In order to protect caregivers, policies and programs designed to promote wellbeing and mitigate the potential harms of caregiving to health should consider these findings. 
Introduction:

Over 34 million American adults have provided informal care to an adult over the age of 50

27 within the last 12 months, $47 \%$ of whom were caring for a parent (National Alliance for Caregiving,

28 2015). These figures are only expected to rise as the number of Americans over 65 years of age grows from 47.5 million in 2015 to 98 million by 2060 (Administration on Aging, 2016). Informal caregiving, the unpaid care and support family members and friends voluntarily provide to

31 individuals who are unable to function independently, has numerous benefits to care recipients and society as a whole. These include savings to the national economy, prevention of hospitalization and institutionalization, and allowing older residents to remain in their own homes (Chari, Engberg, Ray, \& Mehrotra, 2015). Informal caregivers are estimated to have spent over 30 billion hours a year providing care to disabled or chronically ill individuals, with an opportunity cost savings of $\$ 522$ billion per year that would otherwise be spent on formal care and institutionalization (Chari, Engberg, Ray, \& Mehrotra, 2015).

Despite these benefits of informal caregiving to the care recipient and to the national economy, numerous negative effects associated with caregiving have been well documented in the literature. Many studies have demonstrated the negative impacts on caregiver health-related 41 quality of life, including physical and emotional health consequences, that can occur as a result of 42 providing care, such as anxiety and depression (Cannuscio, et al., 2002; Ho, Chan, Woo, Chong, \& 43 Sham, 2009; Macneil et al., 2010; National Alliance for Caregiving, 2015; Pinquart \& Sorensen, 44 2011; Schultz \& Sherwood, 2008). These negative health-related quality of life outcomes are commonly referred to as caregiver stress, strain, or burden. Moreover, informal caregivers providing a high amount of care may be particularly vulnerable to the effects of caregiver strain,

47 and may differ in substantial ways from those providing less care, such as their employment status, 48 the type of caregiving duties they provide, and the impact caregiving has on them (Jacobs, Laporte, 49 Van Houtven, \& Coyte, 2014; National Alliance for Caregiving, 2015). The duties caregivers provide 
may vary by sociodemographic factors, including race and gender, where female and non-white caregivers were more likely to provide higher amounts of informal care than their counterparts (National Alliance for Caregiving, 2015). However findings are mixed. Few studies have examined differences in caregiving intensity by socioeconomic and demographic factors using a nationally representative dataset.

There is a critical need to better understand who is providing informal care to aging parents, and how to best provide the support, assistance, and resources caregivers may need. To further the understanding of who is providing informal care, the objectives of this study were to examine the demographic profile of informal adult-child caregivers in the U.S., and to assess the sociodemographic differences in caregiving duties (caregiving "intensity") among this population of informal caregivers.

\section{Methods:}

Study population

The data were obtained from the 2011 National Study of Caregiving (NSOC) dataset, a nationally representative sample of informal caregivers. The NSOC identified caregivers of National Health and Aging Trends Study (NHATS) participants who were receiving assistance in self-care, mobility, medical, or household activities. These caregivers were then contacted to participate in a one-time, cross-sectional assessment of caregiving that included questions on caregiving activities, duration, intensity, and demographics. This analysis focused on adult children caregivers to older adult parents $(\mathrm{n}=1014)$, a subset of informal caregivers.

\section{Outcome variables: Caregiving intensity}

Four individual measures of caregiving duties that are most common in the literature were used to assess caregiving intensity: 1) Number of Activities of Daily Living (ADLs) performed, 2) Number of Instrumental Activities of Daily Living (IADLs) performed, 3) Hours of caregiving 
provided per month, and 4) Duration (years) of caregiving. To measure these four intensity domains, items from the NSOC questionnaire assessing aspects of caregiver duties were used. ADLs refer to daily self-care activities that are necessary for fundamental functioning. This was measured by the number of personal care activities caregivers helped with each month, including eating, bathing, dressing, toileting, and helping care recipient move around. IADLs consist of other caregiving activities not necessary for fundamental functioning, but allow an individual to live independently. This domain included the number of instrumental activities caregivers helped their parent with, including medication management, scheduling medical appointments, and other health and hygiene-related tasks. The last two intensity domains were calculated based on the average number of hours spent caregiving in the last month, and average number of years providing care. The top quartile (25\%) of each individual intensity domain were considered 'High Intensity Caregivers', while the bottom 75\% were considered 'Low Intensity Caregivers'.

To calculate the composite intensity measure, each of the four individual measures of caregiving intensity scored one point if considered 'high intensity', and zero points if considered "low intensity". This composite score ranged from zero (provided no high intensity care in any of the four individual caregiving measures) to four (provided high intensity care in all four individual caregiver domains).

Exposure variables: Caregiver demographics

Four demographic characteristics of caregiver respondents identified in previous studies of caregiving intensity were assessed, to include caregiver age, gender, race/ethnicity, and annual household income (Navaie-Waliser, Spriggs, \& Feldman, 2002; Navaie-Waliser, et al, 2002; Fredman, Doros, Ensrud, Hochberg, \& Cauley, 2009; Cohen, Cook, Sando, Brown, \& Longo, 2017). Demographic age was categorized into 10-year age groups $(<45,45-54,55-64$, and $65+)$. Race/ethnicity was based on three calculated domains (non-Hispanic White, non-Hispanic Black, and 'Other' (Hispanic, American Indian, Native Hawaiian, Pacific Island, other non-Hispanic)). 
Income was grouped into four $\$ 25,000$ intervals $(\leq \$ 24,999, \$ 25,000-\$ 49,999, \$ 50,000-\$ 74,999$, and $\$ 75,000$ or more).

\section{Additional demographic confounders and covariates}

Other key confounders and covariates commonly used in studies of disparities in caregiver intensity and are shown to affect caregiver intensity, including caregiver marital status (Brody, Litvin, Hoffman, \& Kleban, 1995; Dentinger \& Clarkberg, 2002), presence of a child (under 18) living in the home (Cohen, Cook, Sando, Brown, \& Longo, 2017; Grundy \& Henretta, 2006), and caregiver co-resident status (care recipient and caregiver reside in same home) (Tennstedt, Crawford, \& McKinlay, 1993) were also assessed.

Data analysis

Univariate and bivariate analyses were used for all primary outcome and exposure variables to assess individual measures of high intensity caregiving. For the composite measure of high intensity caregiving, ordinal logistic regression models were used to calculate adjusted odds ratios and 95\% confidence intervals (CI), adjusting for covariates. Pairwise deletion was used to handle missing values for each model. SAS 9.3 (Cary, NC) was used for all analyses.

\section{Results:}

Demographics of NSOC adult-child caregivers

The demographic breakdown for this sample of adult children caregivers is found in Table 1. The average age of caregivers was 54.6 years old. Sixty-nine percent of respondents were female, while $31 \%$ were male. Respondents reported an average annual income of $\$ 56,582$. Sixty percent of respondents identified as non-Hispanic White, 31\% as non-Hispanic Black, and $9 \%$ as another racial/ethnic group. Adult children caregivers spent an average of 85 hours a month providing care, and had been caring for an elderly parent for 5.6 years.

Individual measures of Caregiving Intensity 
High ADL caregiving was most prevalent in caregivers aged 45-54 (28.1\%) and non-

126 Hispanic Black caregivers (33.9\%). High IADL caregiving was significantly higher in females

127 (30.3\%) than in males (20.6\%). Compared to White caregivers, high IADL caregiving was

128 significantly higher among non-Hispanic Black caregivers (33.5\%) and caregivers of 'other'

129 racial/ethnic groups (30.9\%). A high number of hours spent caregiving was highest in females

130 (27.9\%), non-Hispanic Black caregivers (36.1\%) and 'other' race/ethnicities (30.8\%), and

131 individuals earning less than $\$ 25,000$ per year (37.6\%). High years of caregiving was highest in

132 non-Hispanic Black caregivers (33.8\%) and caregivers of 'other' race/ethnicities (35.7\%). All

133 results are displayed in Table 2.

134 Composite measure of caregiving intensity

135 Differences in high intensity caregiving varied by gender, race, and other sociodemographic 136 factors (Figure 1). Female caregivers had higher odds of providing high intensity care than their 137 male caregiver counterparts $(\mathrm{OR}=1.43, \mathrm{CI}[1.03,1.99])$. The odds of providing high intensity 138 caregiving was greater for non-White caregivers (caregivers of Black and 'other' race/ethnicities) 139 than White caregivers (OR $=1.86$, CI $[1.30,2.64])$. Co-resident caregivers were more likely to have 140 provided high intensity caregiving than caregivers not residing with their care recipient $(\mathrm{OR}=1.70$,

141 CI [1.19, 2.42]). Additionally, a negative association between annual income and high intensity 142 caregiving was observed: as caregivers' annual income decreased, the odds of providing high 143 intensity caregiving significantly increased $(\mathrm{p}<0.001)$.

Discussion:

This study sought to better understand who is providing high intensity informal care to an

147 older parent. Our findings indicate that there are notable differences in caregiving intensity that

148 vary by caregiver socioeconomic and demographic factors. High intensity caregiving was most 149 prevalent among females, non-White caregivers, those living with their care recipient, and low 
income caregivers. These results are in line with previous studies that report greater caregiving responsibilities among females, racial/ethnic minorities, shared co-residence, and low income caregivers (Kim, Chang, Rose \& Kim, 2012; Pinquart \& Sörensen, 2005; National Alliance for Caregiving, 2015). However, the observed associations were dependent on the type of care being provided, such that certain caregivers were more likely to provide high intensity care in some domains of caregiving than others. Surprisingly, no differences were observed between males and females in high ADL caregiving. Historically, this has not been the case, and could be a result of the changing face of informal caregivers, or in how the caregivers were selected for inclusion in this sample.

While our results largely confirm previous findings, our analysis adds to the current body of caregiver research in several ways. First, the analysis uses a nationally representative sample of caregivers previously identified by their care recipient. This is an important distinction from other representative samples where respondents self-identify as caregivers. Second, our focus on adult child caregivers sheds light on who is providing high intensity care to an aging parent. Investigating adult child caregivers - an important and large subset of caregivers - is important, as they differ from other types of caregivers (i.e. spousal) in significant ways. Examining this group separately is recommended, as significant differences in caregiver characteristics, needs, and burden have been noted (Chappell, Dujela \& Smith, 2014; Pinquart \& Sörenson, 2011). Third, this analysis compares caregivers based on the level of care they provided (high vs. low intensity). Caregiving intensity, whether measured by the type or amount of assistance provided is associated with various health effects and quality of life outcomes (Shultz \& Sherwood, 2008). Numerous studies have shown any type of informal caregiving can result in negative physical and emotional health consequences for the caregiver, often referred to as caregiving-related stress or burden (Cannuscio, et al., 2002; Ho, Chan, Woo, Chong, \& Sham, 2009; Macneil et al., 2010; National Alliance for Caregiving, 2015; Pinquart \& Sörensen, 2011; Schultz \& Sherwood, 2008). With noted 
175 differences in the intensity of care being provided, we anticipate implications for caregiver health

176 and quality of life that also vary by sociodemographics. Research suggests that differences exist in

177 caregiver quality of life among male and female caregivers, caregivers of different racial and ethnic

178 groups, ages (Neugaard, Andresen, McKune \& Jamoom, 2008; Anderson et al., 2013; Covinsky,

179 2003), and income levels (Williams, Forbes, Mitchell, Essar \& Corbett, 2003).

Health care professionals should be aware of trends in high intensity caregiving among

181 informal caregivers providing high intensity care to an older parent. Gerontological nurses may

182 have frequent contact with aging adults and their informal caregivers who are susceptible to or

183 exhibiting signs of burnout and quality of life concerns. Their role in facilitative informal caregiving

184 has expanded from being primary caregivers to teaching and assisting family members to provide

185 care (Schulz \& Sherwood, 2008). Nurses that provide informal caregivers a temporary break from

186 their caregiving responsibilities can significantly improve caregiver health and quality of life

187 (Lopez-Hartmann, Wens, Verhoeven, \& Remmen, 2012) and reduce subsequent caregiver burden

188 (Horton-Deutsch, Farran, Choi, \& Fogg, 2002).

This group of health providers are especially poised to recognize symptoms of distress and

190 burnout, and offer appropriate resources for caregivers in need of additional supports. Such

191 support is facilitated through communication between nurses and informal caregivers, building

192 relationships with informal caregivers, and creating a culture of trust with the family of the care

193 recipient (Weman \& Fagerberg, 2006). However, the availability of healthcare providers trained to

194 provide such support may depend upon the services and resources that are available at the local

195 level and may not be equitable for all informal caregiver populations throughout the US.

196 Limitations:

When interpreting these findings, there are a few important limitations to note. First, due to

198 the cross-sectional nature of this study, we are unable to determine causal relationships between

199 caregiver demographics and high intensity caregiving. A second wave of NSOC data will be available 


\section{Conclusions: \\ Conclusions:}

within the year and future analyses may be able to determine causality. Second, we did not look at caregiver employment, which may impact caregivers' ability to provide different types and levels of care. Third, caregiving responsibilities was dichotomized into high and low caregiving intensity, rather than assessed along a gradient. As such, a composite analysis was conducted to assess overall caregiving intensity as a continuous measure composed of multiple types of caregiving (e.g. ADLs, IADLs, and hours per month). Next, all measures were self-reported, which may bias the results toward more socially acceptable responses to the measures examined in the study. Sample weights were not used in the analysis, as the importance of including weights in regression models such as these in which descriptive population parameters are not being estimated are a subject of debate in the survey analysis literature. Lastly, we assumed that missing data were missing at random, and therefore did not impute missing values, and instead handled the issue of missing values through the use of pairwise deletion.

Our findings show both females and non-White caregivers are more likely to provide high intensity care, though the intersection between these two constructs and the influence on outcomes is not well known. What remains to be seen is the impact sociodemographic interactions have on caregiver intensity and caregiver health outcomes. In addition, as the number of aging adults increase and the face of caregiving evolves, we anticipate the sociodemographics of caregivers providing high intensity care will also change over time, as well as their needs and necessary supports. Consequently, policies and programs designed to promote caregiver health and quality of life should consider these important sociodemographic disparities to protect and support this vital component of the US health care system. 


\section{References}

Adelman, R.D., Tmanova, L.L., Delgado, D., Dion, S., \& Lachs, M.S. (2014). Caregiver burden a clinical review. JAMA - Journal of the American Medical Association, 311(10), 1052-1059. doi:10.1001/jama.2014.304

Administration on Aging. A Profile of Older Americans: 2016. https://www.giaging.org/documents/A_Profile_of_Older_Americans_2016.pdf

Anderson, L.A., Edwards, V.J., Pearson, W.S., Talley, R.C., McGuire, L.C., \& Andresen, E.M. (2013). Adult caregivers, in the United States: Characteristics and differences in well-being, by Caregiver Age and Caregiving Status. Preventing Chronic Disease, 10, E135. doi: $10.5888 /$ pcd10.130090

Brody, E. M., Litvin, S. J., Hoffman, C., \& Kleban, M. H. (1995). Marital status of caregiving daughters and co-residence with dependent parents. The Gerontologist, 35(1), 75-85.

Cannuscio, C.C., Jones, C., Kawachi, I., Colditz, G.A., Berkman, L., \& Rimm, E. (2002). Reverberations of family illness: A longitudinal assessment of informal caregiving and mental health status in the Nurses' Health Study. American Journal of Public Health, 92(8), 1305-1311.

Chappell, N.L., Dujela, C., \& Smith, A. (2014). Spouse and adult child differences in caregiving burden. Canadian Journal on Aging, 33(4), 462-472. doi: 10.1017/S0714980814000336

Chari, A.V., Engberg, J., Ray, K.N., \& Mehrotta, A. (2015). The opportunity costs of informal eldercare in the United States: New estimates from the American Time Use Survey. Health Services Research, 50(3), 871-882. doi: 10.1111/1475-6773.12238

Cohen, S. A., Cook, S. K., Sando, T. A., Brown, M. J., \& Longo, D. R. (2017). Socioeconomic and Demographic Disparities in Caregiving Intensity and Quality of Life in Informal Caregivers: A First Look at the National Study of Caregiving. Journal of Gerontological Nursing, 43(6), 1724. 
Covinsky, K.E., Newcomer, R., Fox, P., Wood, J., Sands, L., Dane, K., \& Yaffe, K. (2003). Patient and caregiver characteristics associated with depression in caregivers of patients with dementia. Journal of General Internal Medicine, 18(12), 1006-1014.

Dentinger, E., \& Clarkberg, M. (2002). Informal caregiving and retirement timing among men and women: Gender and caregiving relationships in late midlife. Journal of Family Issues, 23(7), 857-879.

Fredman, L., Doros, G., Ensrud, K. E., Hochberg, M. C., \& Cauley, J. A. (2009). Caregiving intensity and change in physical functioning over a 2-year period: results of the caregiver-study of osteoporotic fractures. American Journal of Epidemiology, 170(2), 203-210.

Grundy, E., \& Henretta, J. C. (2006). Between elderly parents and adult children: A new look at the intergenerational care provided by the 'sandwich generation'. Ageing \& Society, 26(5), 707722.

Ho, S.C., Chan, A., Woo, J., Chong, P., \& Sham, A. (2009). Impacts of caregiving on health and quality of life. Journal of Gerontology: Series A Biological Sciences \& Medical Sciences, 64A(8), 873879. doi:10.1093/gerona/glp034

Horton-Deutsch, S. L., Farran, C. J., Choi, E. E., \& Fogg, L. (2002). The PLUS intervention: A pilot test with caregivers of depressed older adults. Archives of Psychiatric Nursing, 16(2), 61-71.

Jacobs, J.C., Laporte, A., Van Houtven, C.H., \& Coyte, P.C. (2014). Caregiving intensity and retirement status in Canada. Social Science \& Medicine, 102(2014), 74-82. doi:10.1016/j.socscimed.2013.11.051

Kim, H., Chang, M., Rose, K., \& Kim, S. (2012). Predictors of caregiver burden in caregivers of individuals with dementia. Journal of Advanced Nursing, 68(4), 846-855.

Lopez-Hartmann, M., Wens, J., Verhoeven, V., \& Remmen, R. (2012). The effect of caregiver support interventions for informal caregivers of community-dwelling frail elderly: A systematic review. International Journal of Integrated Care, 12. 
272 Macneil, G., Kosberg, J.I., Durkin, D.W., Dooley, W.K., Decoster, J., \& Williams, G.M. (2010). Caregiver mental health and potentially harmful caregiving behavior: The central role of caregiver anger. The Gerontologist, 50(1), 76-86. doi: 10.1093/geront/gnp099

National Alliance for Caregiving (NAC) and AARP. Research Report: Caregiving in the U.S. 2015 - A Focused Look at Caregivers of Adults Age 50+. (June 2015). http://www.aarp.org/content/dam/aarp/ppi/2015/caregivers-of-older-adults-focusedlook.pdf

Navaie-Waliser, M., Feldman, P. H., Gould, D. A., Levine, C., Kuerbis, A. N., \& Donelan, K. (2002). When the caregiver needs care: The plight of vulnerable caregivers. American Journal of Public Health, 92(3), 409-413.

Navaie-Waliser M, Spriggs A, Feldman P.H. (2002). Informal caregiving: Differential experiences by gender. Medical Care, 40(12), 1249-1259.

Neugaard, B., Andresen, E., McKune, S.L., \& Jamoom, E. (2008). Health-Related quality of life in a national sample of caregivers: Findings from the Behavioral Risk Factor Surveillance System. Journal of Happiness Studies, 9(4), 559-575. doi: 10.1007/s10902-008-9089-2

Pinquart, M., \& Sörensen, S. (2005). Ethnic differences in stressors, resources, and psychological outcomes of family caregiving: A meta-analysis. The Gerontologist, 45(1), 90-106.

Pinquart, M. \& Sörensen, S. (2011). Spouses, adult children, and children-in law as caregivers of older adults: a meta-analytic comparison. Psychology and Aging, 26(1), 1-14. doi: $10.1037 / \mathrm{a} 0021863$

Schultz, R., \& Sherwood, P.R. (2008). Physical and mental health effects of family caregiving. American Journal of Nursing, 108(9), 23-27. doi: 10.1097/01.NAJ.0000336406.45248.4c

Tennstedt, S. L., Crawford, S., \& McKinlay, J. B. (1993). Determining the pattern of community care: Is coresidence more important than caregiver relationship? Journal of Gerontology, 48(2), S74-S83. 
297 Weman, K., \& Fagerberg, I. (2006). Registered Nurses working together with family members of $298 \quad$ older people. Journal of Clinical Nursing, 15(3), 281-289.

299 Williams, A.M., Forbes, D.A., Mitchell, J.M., Essar, M., \& Corbett, B. (2003). The influence of income on 300 the experience of informal caregiving: Policy implications. Health Care for Women

301 International, 24(4), 280-291. doi: 10.1080/07399330390183606 